\title{
Refractory Longitudinally Extensive Transverse Myelitis Responsive to Cyclophosphamide
}

\author{
Laura J. Baxter, Shuo Chen, Philippe Couillard, James N. Scott, Christopher J. Doig, \\ Fiona Costello, Louis P. Girard, John Klassen, Jodie M. Burton
}

\begin{abstract}
Severe longitudinally extensive transverse myelitis (LETM) can cause quadriplegia, marked sensory dysfunction, and respiratory failure. Some patients are unresponsive to conventional immune therapy. We report two cases of severe immune-mediated LETM requiring intensive care admission that failed to respond to high-dose corticosteroids, plasma exchange, intravenous immunoglobulin, and rituximab. Disease cessation and significant recovery was achieved after cyclophosphamide induction. In patients with severe acute immune-mediated LETM who fail to respond to corticosteroids and plasma exchange, cyclophosphamide induction should be considered. This agent and regimen provides a robust immunosuppressive response and can be induced rapidly. Cyclophosphamide effects and supportive evidence are discussed.
\end{abstract}

RÉSUMÉ: Myélite transverse longitudinalement étendue répondant à la cyclophosphamide. La myélite transverse longitudinalement étendue (MTLE) peut causer une quadriplégie, une dysfonction sensitive importante et une insuffisance respiratoire. Certains patients ne répondent pas à l'immunothérapie conventionnelle. Nous rapportons deux cas de MTLE sévère d'origine auto-immune nécessitant une hospitalisation à l'unité des soins intensifs et ne réagissant pas au traitement par les corticostéroïdes à haute dose, à la plasmaphérèse, à l'immunoglobuline intraveineuse ou au rituximab. Le traitement par la cyclophosphamide a entraîné l'arrêt de la maladie et une récupération importante. Chez les patients qui présentent une MTLE autoimmune aiguë sévère qui ne répond pas aux corticostéroïdes et à la plasmaphérèse, le traitement par la cyclophosphamide devrait être envisagé. Cet agent provoque une réponse immunosuppressive importante qui peut être induite rapidement. Nous discutons des effets de la cyclophosphamide et des données appuyant son utilisation.

Keywords: transverse myelitis, cyclophosphamide, neuroimmunology

doi:10.1017/cjn.2017.201

Can J Neurol Sci. 2017; 44: 736-739

Longitudinally extensive transverse myelitis (LETM) describes myelitis spanning three or more vertebral segments ${ }^{1,2}$ that can cause quadriplegia, marked sensory dysfunction, autonomic instability, and respiratory failure in severe cases. ${ }^{3}$ It is associated with many causal entities, including neuromyelitis optica spectrum disorder (NMOSD), postinfection/vaccination, acute demyelinating encephalomyelitis, lymphoma, paraneoplastic conditions, and systemic autoimmune processes such as systemic lupus erythematosus and Sjögren's syndrome. ${ }^{1}$ We report two severe LETM cases that were refractory to multiple conventional immunotherapies, including high-dose corticosteroids, plasmapheresis (PLEX), and intravenous immunoglobulin. In both cases, meaningful recovery was only achieved after cyclophosphamide induction.

In case 1, a 21-year-old, previously healthy female presented to hospital with a 5-day history of hiccups, abdominal pain, paresthesias, pruritus, and rash. Three weeks prior, she had a brief, nonspecific flulike illness. Other than mild sinus tachycardia and maculopapular rash on her right chest and posterior neck, her systemic examination was normal. Neurologic examination revealed no abnormalities. After admission to internal medicine for suspected viral infection, the neurology consult team recommended a brain and cervical spine magnetic resonance imaging (MRI) scan given her symptoms.

In hospital, abdominal and pelvic ultrasound was noncontributory. Brain MRI was unremarkable, but the suggested spinal MRI was not performed. Over the next 4 days, paresthesias became generalized with accompanying allodynia and urinary retention. On day 5, rapidly ascending symmetric leg weakness developed (Medical Research Council [MRC] Muscle scale, 3 to 4-). Urgent complete spinal MRI demonstrated an edematous T2 hyperintensity extending from the cervical to lumbar spine, with gadolinium enhancement (Figure 1A-D). Repeat brain MRI scan showed new subtle periventricular white matter lesions and a large cervicomedullary junction lesion (Figure 1A-B). Urgent lumbar puncture revealed elevated protein $0.85 \mathrm{~g} / \mathrm{l}$, white blood cell count $133.3 \times 10^{6} / \mathrm{L}$ (92\% lymphocytes), and oligoclonal banding was positive. Intravenous (IV) methylprednisolone $1 \mathrm{~g} /$ day for 6 days

From the Department of Clinical Neurosciences, Faculty of Medicine, University of Calgary, Calgary, Alberta (LJB, SC, PC, JNS, FC, JMB); Department of Critical Care Medicine, Faculty of Medicine, University of Calgary, Calgary, Alberta (PC, CJD); Department of Diagnostic Imaging, Faculty of Medicine, University of Calgary, Calgary, Alberta (JNS); Department of Surgery, Faculty of Medicine, University of Calgary, Calgary, Alberta (CJD, FC); Division of Nephrology, Faculty of Medicine, University of Calgary, Calgary, Alberta (LPG, JK); Department of Pathology and Laboratory Medicine, University of Calgary, Calgary, Alberta (JK); Department of Community Health Sciences, University of Calgary, Calgary, Alberta (JMB).

Received June 29, 2016. Final Revisions Submitted March 27, 2017. Date of ACCEPTANCE APRIL 2, 2017.

Correspondence to: Laura J. Baxter, Adult Neurology Residency Program Office,

Department of Clinical Neurosciences, 12th Floor, Foothills Medical Centre, 1403 - 29 Street NW, University of Calgary, Calgary, AB T2N 2T9. Email: laura.baxter@ahs.ca 
was started on day 8. Positive serum and cerebral spinal fluid aquaporin-4 (AQP4) antibodies confirmed a diagnosis of NMOSD. While on corticosteroids, dysarthria, diplopia, nystagmus, and hypoxemic respiratory failure developed, requiring intubation. IV immunoglobulin $(2 \mathrm{~g} / \mathrm{kg}$ total $)$ was added on day 9 , but deterioration persisted until quadriplegia and total anesthesia developed. Thus, plasma exchange (PLEX) was initiated on day 13. After eight exchanges with no improvement, rituximab induction $\left(375 \mathrm{mg} / \mathrm{m}^{2}\right.$ every week for 4 weeks) was added on day 19. ${ }^{4}$ Despite a total of 25 exchanges and rituximab induction, she had no clinical improvement. Repeat MRI scan showed reduction in cord expansion and size of her spinal lesions, but brain imaging was unchanged.

Given her lack of clinical improvement, cyclophosphamide induction was initiated on day 52 whilst continuing PLEX. This consisted of $600 \mathrm{mg} / \mathrm{m}^{2}$ IV cyclophosphamide on days 1, 2, 4, 6, and 8 with daily methylprednisolone (modification of the Northeast Cooperative Trial protocol). ${ }^{5,6}$ Although neutropenia necessitated cyclophosphamide discontinuation after two doses, her neurological function improved within 5 days. Two weeks later, she left the intensive care unit (ICU) after a 63-day stay. At that time, she had substantial improvement in arm strength (MRC 4-5), but remained paraplegic. PLEX was discontinued 1 month after cyclophosphamide induction (total of 40 exchanges administered). Maintenance therapy with azathioprine (3-week titration to $150 \mathrm{mg}$ /day divided in three doses) and a slow prednisone taper $(60 \mathrm{mg} /$ day titrated down to discontinuation over 10 months) was initiated within weeks of completing cyclophosphamide. After 5 months of inpatient rehabilitation, she regained full arm strength and sufficient leg strength to ambulate with a walker (MRC 3-4). At 11 months, she had MRC 5 power throughout, except for right ankle dorsiflexion (MRC 2).

In case 2, a 19-year-old male with well-controlled celiac disease presented with rapid-onset anesthesia, quadriplegia, and respiratory failure requiring intubation and ICU admission in less than 24 hours. Three days prior, he had a cough and sore throat, but no fever. Three weeks prior, he had an episode of nonbloody diarrhea and a suspected tick bite. On initial assessment, he was intubated, but alert with intact comprehension. He had gaze-evoked nystagmus, mild bifacial weakness, flaccid quadriplegia sparing only the right finger flexors (MRC 1), and a T3 sensory level. Deep tendon reflexes were absent except for $1+$ right ankle jerk.

After normal routine serology and cerebral spinal fluid with no evidence of infection, IV methylprednisolone $1 \mathrm{~g}$ /day for 5 days was initiated on admission. Brain MRI was unremarkable, but cervical and thoracic spine MRI demonstrated hyperintensity in the central cord and subtle gadolinium enhancement from $\mathrm{C} 2-\mathrm{C} 5$ (Figure 2A-B). Given clinical severity, PLEX was started on day 2. Testing for infectious (including tickborne), metabolic, neoplastic, and autoimmune abnormalities including oligoclonal banding, AQP4, myelin oligodendrocyte glycoprotein, and paraneoplastic antibodies were negative, making the working diagnosis isolated AQP4- LETM.

Repeat MRI (Figure 2C-D) on day 3 of PLEX showed worsening cord edema and increased enhancement. Sensory level on examination had ascended to $\mathrm{C} 4$. After 14 plasma exchanges, clinical status remained unchanged, and LETM had spread to $\mathrm{C} 1-\mathrm{C} 6 / 7$ on MRI (Figure 2E-F), with further increase in enhancement. Given his refractory clinical status and worsening MRI (despite PLEX and high-dose corticosteroids), cyclophosphamide induction (as described in case 1) was completed on day $16^{5,6}$ Within a week of induction, motor function began to improve rapidly. Repeat MRI (Figure 2G-H) 2 weeks later showed resolving cord edema and resolution of enhancement. One month later, he was discharged from the ICU and quickly transferred to inpatient neurorehabilitation where he continued a 4-month oral prednisone taper. No further immunosuppressive or immunomodulatory therapy was given. After 5 months of rehabilitation, he was discharged home. He had regained bladder and bowel control, and walked with a walker and ankle foot orthotic. Right deltoid was MRC 2, right bicep MRC 3, bilateral knee flexion MRC 3, left ankle flexion MRC 2, and the remainder of his power examination was MRC 4 or greater.

LETM is a variable entity seen in several conditions, two of which (NMOSD and isolated AQP4- LETM) are presented here. ${ }^{1}$ Although most patients respond to first-line therapies of high-dose

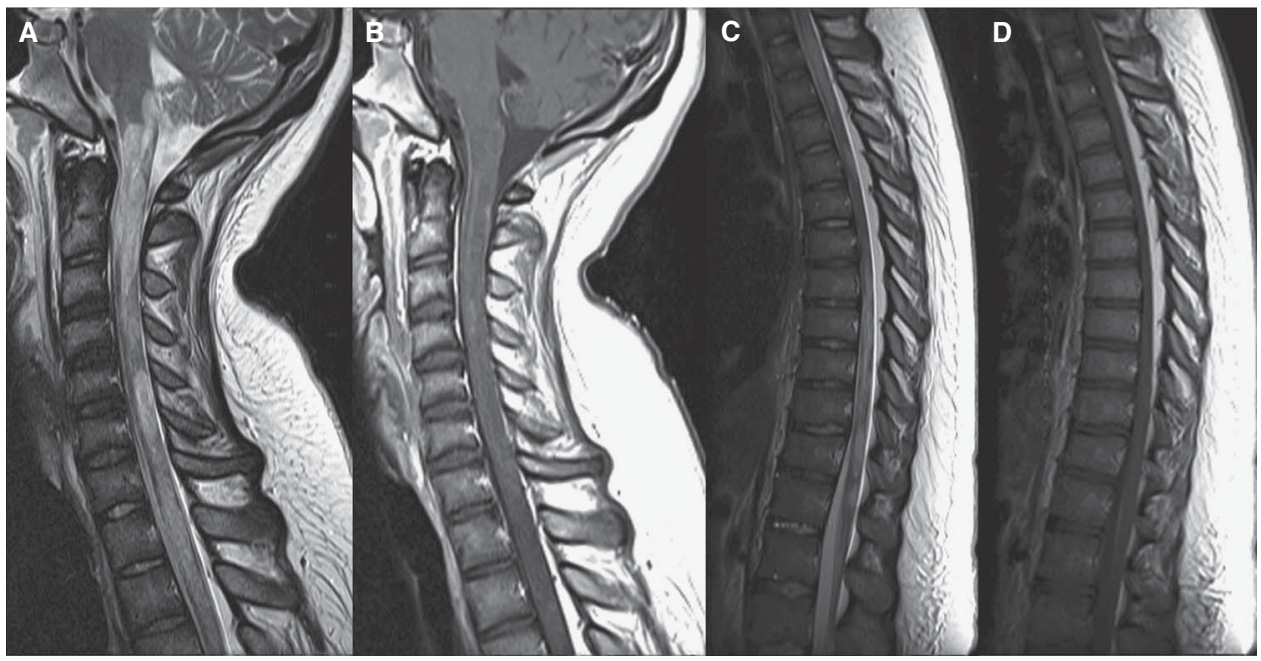

Figure 1: Sagittal MRI scan of the cervical and thoracic spine in case 1. Sagittal T2 of the cervical (A) and thoracic $(C)$ spine shows extensive hyperintensity and areas of fusiform enlargement extending from the lower brainstem throughout the spinal cord and conus medullaris. Some involved areas demonstrate mild patchy enhancement on postgadolinium $T 1$ images $(B, D)$. 

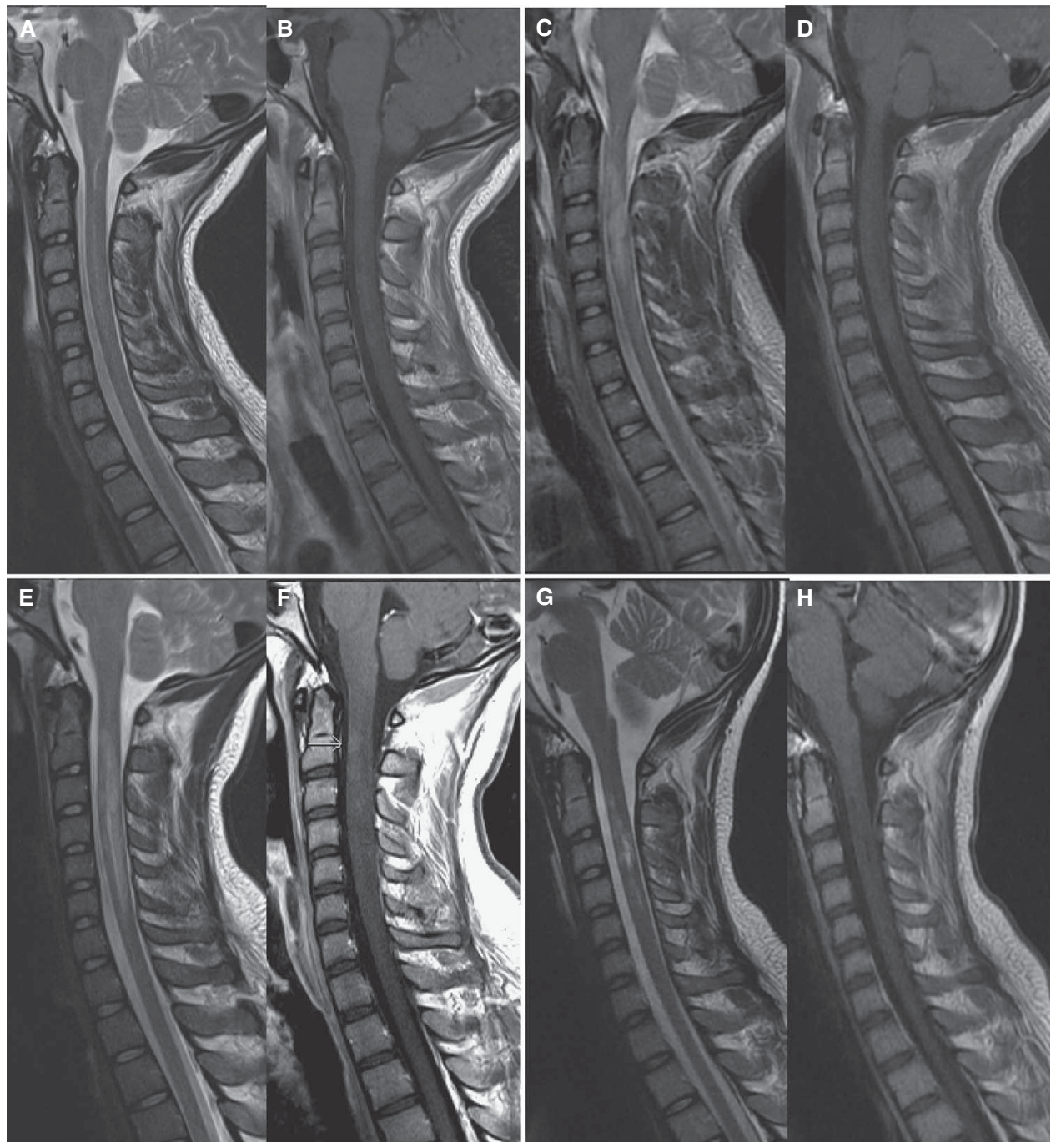

Figure 2: Sagittal MRI scan of the cervical spine in case 2. On admission, sagittal MRI of the cervical spine shows hyperintensity of the central spinal cord from C2-C5 levels on T2 images (A) with subtle corresponding enhancement on postgadolinium T1 images (B). After 4 days of high-dose IV methylprednisolone and three plasma exchanges, repeat MRI shows increased edema and hyperintensity on $\mathrm{T2}$ images from $\mathrm{C2}-\mathrm{C5} \quad(\mathrm{C})$ with cord expansion, and progressive cord enhancement on postgadolinium images (D). After completing a 5-day course of high-dose IV methylprednisolone and 14 plasma exchanges, repeat MRI shows further extension of cord involvement from C2-C6/7 levels with T2 hyperintensity $(E)$ and increased enhancement on postgadolinium $T 1$ images $(F)$. Two weeks after completion of cyclophosphamide induction, repeat MRI shows resolving cord edema on T2 images $(G)$ and resolution of cord enhancement on postgadolinium $T 1$ images $(H)$.

corticosteroids and PLEX, a minority of patients do not. In case 1, the NMOSD patient failed high-dose corticosteroids, IVIg, PLEX, and rituximab before cyclophosphamide induction. Rituximab is not known to be an acute rescue therapy for NMOSD; rather, it is considered a longer term disease-modifying therapy. ${ }^{7}$ If rituximab were to exert early clinical effects, results would be expected within 1 to 2 weeks as CD20 B-cell depletion occurs within this time frame, which did not occur in case $1 .{ }^{8}$ One or both treatments likely contributed to her radiographic improvement, which was seen before cyclophosphamide. Rituximab may have contributed to long-term recovery; however, neither PLEX nor rituximab, both antibody-targeted therapies, provided any acute clinical improvement. In case 2, the isolated LETM patient worsened clinically and radiographically despite high-dose corticosteroids and PLEX.
Although no standardized PLEX duration exists, interstitial immunoglobulin $\mathrm{G}$ depletion can be expected within approximately five exchanges. ${ }^{9}$ Both patients received much longer PLEX courses without clinical benefit. Both patients had neurologic improvement within days of cyclophosphamide. Their rapid clinical response suggests cyclophosphamide contributed to their acute recovery, expediting ICU discharge and rehabilitation.

Cyclophosphamide has long been used as an off-label rescue therapy for refractory immune-mediated transverse myelitis; however, no randomized control trials exist to provide high level evidence for such use. ${ }^{10}$ One study of 122 patients with idiopathic transverse myelitis compared clinical outcomes of corticosteroid treatment, PLEX, cyclophosphamide (750 to $1,000 \mathrm{mg} / \mathrm{m}^{2}$ ), or PLEX plus cyclophosphamide. ${ }^{3}$ Severe cases (American Spinal 
Injury Association, level $\mathrm{A}^{11}$ ) who received either cyclophosphamide, or PLEX and cyclophosphamide, had better recovery than those receiving corticosteroids or PLEX alone. However, many patients initially labelled as idiopathic in this study were later found to have underlying autoimmune diseases. Treatment groups were heterogeneous, making comparisons between groups difficult. Nonetheless, this study suggests cyclophosphamide is useful in severe acute LETM cases with varied immunologic backgrounds.

The few reports of cyclophosphamide in NMOSD are conflicting. In a series of four patients with AQP4 + NMOSD, cyclophosphamide treatment of variable doses $\left(500-774 \mathrm{mg} / \mathrm{m}^{2}\right.$ ) was associated with functional improvement. ${ }^{12}$ Another series of seven patients with NMOSD given pulse cyclophosphamide and methylprednisolone found only one patient was clinically stabilized $^{13}$; however, only one patient was AQP4 + in this case series because of limited antibody testing availability.

Our two cases suggest high-dose cyclophosphamide is beneficial in acute refractory LETM. Presumably, this is due to its rapid immunoablative effect with high-dose induction regimens. Contributory effects of PLEX are possible, but lack of clinical improvement despite multiple exchanges suggests it provided little benefit. Although rituximab likely contributed to long-term recovery in case 1 , it is not considered an acute rescue therapy. Rapid improvement shortly after cyclophosphamide induction supports its role the acute setting.

Severe acute LETM requires aggressive treatment as the alternative is often neurologically devastating, with prolonged ICU stays and ventilator dependency. Larger studies are needed to further guide therapeutic decisions and confirm efficacy of specific immune therapies in the critical care setting. However, in patients who fail corticosteroids and PLEX, cyclophosphamide induction should be considered their acute course, not only to promote early neurological recovery, but to reduce ICU admission time and nosocomial complications.

\section{ACKNOWLedgments AND Funding}

None.

\section{Disclosures}

JMB has the following disclosures: Novartis, consultant/ advisor, honoraria/consulting fees; Sanofi Genzyme, consultant/ advisor, honoraria; TEVA, consultant/advisor, honoraria; Biogen, consultant/advisor, honoraria; EMD Serono, consultant/advisor, honoraria. The remaining authors do not have anything to disclose.

\section{Statement of Authorship}

JMB prepared and wrote case 2 as well as the abstract, introduction, discussion, and prepared revisions. SC prepared and wrote case 1 , and contributed to parts of the written discussion. JMB guided the selection of appropriate patients and oversaw revisions. All other authors offered their expertise and contributed to editing.

\section{REFERENCES}

1. Greenberg BM, Frohman EM. Immune-mediated myelopathies. Continuum. 2015;21:121-31.

2. Scott TF, Frohman EM, De Seze J, Gronseth GS, Weinshenker BG. Evidence-based guideline: clinical evaluation and treatment of transverse myelitis. Neurology. 2011;77:2128-34.

3. Greenburg GM, Thomas KP, Krishnan C, Kaplin AI, Calabresi PA, Kerr DA. Idiopathic transverse myelitis: Corticosteroids, plasma exchange, or cyclophosphamide. Neurol. 2007;68: 1614-7.

4. Cree BA, Lamb S, Morgan K, Chen A, Waubant E, Genain C. An open label study of the effects of rituximab in neuromyelitis optica. Neurology. 2005;64:1270-2.

5. Weiner HL, Mackin GA, Orav EJ, et al. Intermittent cyclophosphamide pulse therapy in progressive multiple sclerosis: final report of the Northeast Cooperative Multiple Sclerosis Treatment Group. Neurology. 1993;43:910-8.

6. Partners MS. Center. Cytoxan/methylprednisolone in-patient induction regimen physician's orders. Available from: http:// partnersmscenter.org/index.php?id $=45 \& \mathrm{mn}=5 \& \mathrm{sm}=5-4$.

7. Kim S-H, Huh S-Y, Lee SJ, Joung A, Kim HJ. A 5-year follow-up of rituximab treatment in patients with neuromyelitis optica spectrum disorder. JAMA Neurol. 2013;70:1110-7.

8. Stasi R, Pagano A, Stipa E, Amadori S. Rituximab chimeric anti-CD20 monoclonal antibody treatment for adults with chronic idiopathic thrombocytopenic purpura. Blood. 2001;98: 952-7.

9. Ward DM. Conventional apheresis therapies: a review. J Clin Apher. 2011;26:230-8.

10. Sackett DL. Rules of evidence and clinical recommendations on the use of antithrombotic agents. Chest. 1989;95:2S-4S.

11. International Standards for Neurological Classification of SCI (ISNCSCI) Exam. Available from: http://asia-spinalinjury. org/wp-content/uploads/2016/02/International_Stds_Diagram_ Worksheet.pdf.

12. Yaguchi H, Sakushima K, Takahashi I, et al. Efficacy of intravenous cyclophosphamide therapy for neuromyelitis optica spectrum disorder. Intern Med. 2013;52:969-72.

13. Bichuetti DB, Oliveira EM, de Castro Boulos Fde C, Gabbai AA. Lack of response to pulse cyclophosphamide in neuromyelitis optica: evaluation of 7 patients. Arch Neurol. 2012;69: 938-9. 